\title{
Le récepteur du TGF- $\beta$, cible des anomalies de réparation dans des formes congénitales du cancer colorectal non polyposique
}

\begin{abstract}
Quatre types d'anomalies de gènes de réparation intervenant dans la détection et l'élimination des mésappariements sont en cause dans les formes non polyposiques du cancer colorectal familial [1]. Le phénotype associé à ces anomalies de réparation est dit RER pour repair of errors. Le mécanisme supposé de la sensibilité au cancer des malades atteints est la mutation fréquente d'un gène dont l'activation (dans le cas d'un oncogène) ou l'inactivation (dans le cas d'un anti-oncogène) peut entraîner un avantage prolifératif des cellules ainsi modifiées. Les malades atteints des formes familiales héritent d'un gène muté, et sont donc hétérozygotes. Cependant, le déficit se complète par perte d'hétérozygotie au niveau de la tumeur qui apparaît ainsi totalement déficiente en enzyme codée par le gène muté. Naturellement, les défauts de réparation sont maximaux dans ces cellules tumorales, mais ils sont également détec-
\end{abstract} tables dans les cellules normales [2]. La question fondamentale dont la réponse était anxieusement attendue était celle des gènes-cibles du processus mutagène, c'est-à-dire des gènes dont la mutation non réparée pouvait entraîner l'apparition du cancer, ou au moins intervenir dans la progression tumorale. Plusieurs équipes américaines associées, de Cleveland $(\mathrm{OH})$, Toledo $(\mathrm{OH})$ et Baltimore (MD), apportent une première réponse, qui pourrait être essentielle, voire définitive: le récepteur de type II du TGF- $\beta$ est une cible privilégiée des mutations chez les malades atteints de ces déficits des systèmes de réparation des mésappariements [3]. Trente-huit lignées de cellules étudiées, onze avec un phénotype $\mathrm{RER}^{+}$(c'est-à-dire avec déficit des systèmes de réparation) et vingt-sept avec un phénotype RER ${ }^{-}$. Neuf des onze lignées $\mathrm{RER}^{+}$ont une concentration diminuée du messager codant pour le récepteur de type II (RII) du TGF- $\beta$ alors que ce phénomène n'est noté que trois fois dans les vingt-sept lignées $\mathrm{RER}^{-} \mathrm{Si}$ maintenant l'étude porte sur des cellules tumorales greffées à des souris athymiques nude, la diminution de l'abondance du messager RII est notée dans trois cas sur vingt sept, correspondant tous à des phénotypes RER+. Le déficit est également retrouvé au niveau fonctionnel, en étudiant la liaison à la membrane du TGF- $\beta$ marqué à l'iode 125 . Un déficit fonctionnel est même noté, une fois, en l'absence de diminution de l'abondance du messager. Dans ce cas, est observée une insertion d'un doublet GT dans une séquence hexanucléotidique GTGTGT, provoquant un décalage de phase de lecture et engendrant l'apparition d'un codon stop prémature. Dans sept cas avec diminution de la quantité du messager, la séquence de la région 5' est déterminée. En effet, il est connu que des mutations nonsens situées en 5' des ARN messagers aboutissent à des réductions de leur stabilité. Dans tous les cas, les auteurs trouvent une mutation portant sur une série de dix adénines qui se suivent: délétion d'une base dans quatre cas et de deux bases dans trois cas, aboutissant donc, dans l'une et l'autre situation, à un décalage de phase de lecture avec les conséquences discutées plus haut.

Les lignées de cellules tumorales ayant un récepteur du TGF- $\beta$ normal sont sensibles à ce facteur de crois- sance qui diminue la prolifération cellulaire alors que les lignées déficientes ne répondent pas au TGF- $\beta$. L'introduction dans une lignée déficiente d'un vecteur d'expresion codant pour un récepteur RII fonctionnel permet de restituer l'inhibition par le TGF- $\beta$. Le TGF- $\beta$ est connu depuis longtemps comme un inhibiteur de la croissance des cellules épithéliales et la diminution de cette inhibition a déjà été corrélée à des processus tumoraux, notamment dans le cancer du sein. Dans ce dernier cas, en effet, les oestrogènes semblent capables de diminuer la synthèse de TGF- $\beta$, expliquant ainsi, peut-être, l'augmentation de la prolifération cellulaire.

Ces résultats sont tout à fait spectaculaires et remarquables, constituant une suite brillante et logique aux travaux sur l'association entre les cancers coliques familiaux non polyposiques et les anomalies des gènes de réparation des mésappariements. En effet, ces anomalies de réparation sont associées à la mutation d'un gène de contrôle de la prolifération cellulaire. Ce gène peut être considéré comme un "anti-oncogène": il code en effet pour le récepteur d'un facteur diffusible à effet anti-prolifératif. L'association entre les anomalies de réparation des mésappariements et la mutation du récepteur RII du TGF- $\beta$ paraît très forte, au niveau des tumeurs coliques primitives aussi bien que des lignées cellulaires établies. Le mécanisme de la mutation semble, dans les huit cas testés, extrêmement clair : il est directement relié à l'instabilité des séquences microsatellites grâce à laquelle a été découvert le phénotype RER. En effet, dans tous les cas, la 
mutation est un décalage de phase de lecture dû à une modification du nombre d'éléments répétés, au niveau d'un micro-micro-satellite GT en 3' du messager, ou, beaucoup plus souvent, au niveau d'un minimicro-satellite poly A en 5'. Dans ce dernier cas, le décalage précoce de phase de lecture entraîne une instabilité du messsager, et donc la diminution de son abondance dans les cellules tumorales.

A. $\mathbf{K}$.

1. Thomas (; Dix ans de recherche sur les prédispositions génétiques au développement des tumeurs. médecine/sciences 1995; $11: 336-48$.

2. Markowitz S, Wang J, Myeroff L, Parsons R, Sun L, Lutterbaugh J, Fan RS, Zborowska E, Kinzler $\mathrm{KW}$, Vogelstein B, Brattain M, Willson, JKV. Inactivation of the type $11 \mathrm{TC} \cdot \mathrm{F}-\beta$ receptor in colon cancer cells with microsatellite instability. Science $1995 ; 268: 1336-8$.

3. Parsons R, Li GM, Longley M, Modrich P, Liu B, Berk T, Hamilton SR, Kinzler, KW, Vogelstein B. Mismatch repair deficiency in phenotypically normal human cells. Science 1995; 268: 738-40.

\section{BRÈVES}

$\square$ Pronostic d'un paludisme sévère au lit du malade chez l'enfant africain. Le paludisme à $P$. falciparum reste une cause majeure de mortalité chez les enfants africains. La prise en charge en est difficile, car il s'agit d'une urgence à résoudre au lit du malade, les examens de laboratoire n'étant souvent ni disponibles ni fiables. Des critères de sévérité définis par l'OMS doivent être simplifiés : ils se réfèrent à un nombre trop élevé de symptômes pour être facilement utilisables et, par ailleurs, l'expression clinique du paludisme varie avec l'âge du malade et le niveau d'endémicité. Une équipe anglaise de chercheurs et de cliniciens a mené dans ce but une étude prolongée au Kenya [1]. Dans un hôpital de district, pendant deux ans et demi, 1844 enfants ont été admis avec un diagnostic de paludisme confirmé par la parasitémie, aucune autre cause n'expliquant le syndrome morbide. Examinés avec les critères de l'OMS, ils ont été traités selon un protocole défini ; l'évolution a ensuite été analysée en fonction des signes cliniques et de données biologiques disponibles. Deux

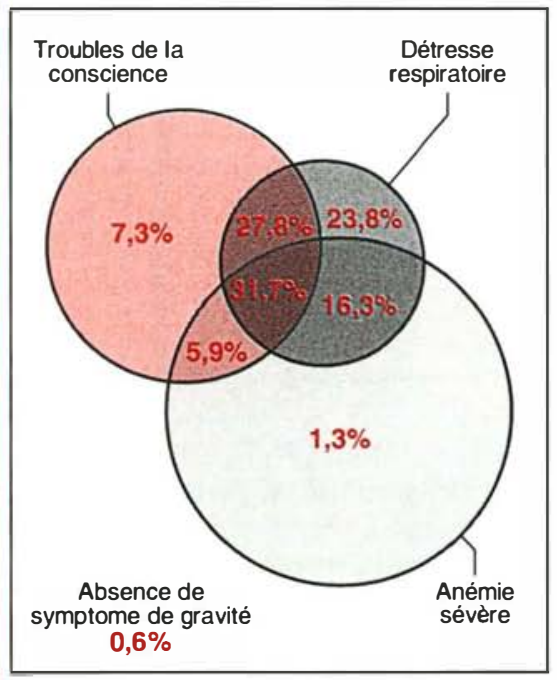

séries de symptômes sont largement dominants comme indices de gravité, justifiant une attention et un traitement particuliers. Une symptomatologie neurologique, troubles de la conscience ou prostration tels que l'enfant ne se maintient pas assis, est décrite depuis longtemps dans le paludisme cérébral grave. Moins connus, et observés surtout chez les enfants les plus jeunes (âge moyen : 19 mois), sont les signes de détresse respiratoire, battement des ailes du nez et tirage intercostal, qui, plus que la tachypnée ou les signes d'auscultation, sont de mauvais pronostic et traduisent la défaillance cardiaque et une acidose métabolique sévère. L'anémie, même profonde mais isolée, serait moins grave. La mortalité en fonction des différents symptômes est schématisée sur la figure 1. L'intérêt de cette étude est de fournir des indices cliniques immédiatement utilisables, plus simples et aussi informatifs que ceux qui sont préconisés par l'OMS.

[1. Marsh K, et al. N Engl J Med 1995 ; 332 : 1399-404.]

Figure 1. Représentation schématique de l'évolution du paludisme chez les enfants africains faisant l'objet de la présente étude. Par rapport à la cohorte complète, les différents cercles représentent la prévalence des trois séries de complications considérées comme graves, à l'état isolé ou associé. Les chiffres rouges indiquent le taux de mortalité, en général dans les 24 premières heures, en fonction de ces complications. (D'après [1].) 


\section{BRÈVES}

Un permis de se répliquer aux règles strictes. Le maintien de l'état diploïde des cellules exige que l'ADN ne se réplique qu'une seule fois par cycle cellulaire. Comment est assurée cette étroite coordination entre la synthèse d'ADN et la division des cellules? Il y a déjà quelques années qu'a été impliquée l'action d'un facteur permissif à la réplication (RLF, replication licencing factor) qui serait responsable de cette synchronisation. Le RLF, cytoplasmique, ne pourrait se lier à la chromatine qu'à l'occasion de la rupture de la membrane nucléaire au cours de la division cellulaire et, après reformation des noyaux, resterait fermement associé à la chromatine et serait indispensable à la réplication de l'ADN. Cependant, cette réplication aboutirait à la dissociation du RLF, interdisant par conséquent une nouvelle réplication de l'ADN avant que la chromatine ne se soit rechargée en ce complexe à l'occasion de la division cellulaire suivante. Les protéines MCM (minichromosome maintenance) étaient de bonnes candidates pour participer à l'activité du RLF. Comme leur nom l'indique, ces protéines sont indispensables à la stabilité des minichromosomes chez la levure. Elles sont localisées dans le noyau de cet organisme après la mitose mais sont absentes après la synthèse d'ADN, c'est-à-dire en phase G2. Des homologues de ces protéines MCM ont été trouvés chez les mammif ères. Des anticorps antiMCM bloquent la synthèse d'ADN chez des vertébrés. Quoique restant nucléaires, les MCM de mammifères sont fortement liées à la chromatine en Gl mais n'y sont plus associées en G2. Trois laboratoires démontrent maintenant sans ambiguïté que des facteurs MCM sont bien des composants essentiels du RLF [1-3]. Chong et al., de Herts et Cambridge, en Angleterre, utilisent un dosage fonctionnel du RLF pour purifier les facteurs actifs. Ce dosage consiste à mettre des noyaux de spermatozoïdes de xénope débarrassés de leur membrane au contact de l'extrait à tester. La préparation purifiée comporte plusieurs fractions dont l'une, homogène, est l'équivalent de la protéine MCM3 chez le xénope [1]. Madine et al. (Cambridge, Angleterre) [2] et Kubota et al. (Osaka, Japon) [3] montrent que des anticorps dirigés contre MCM3 bloquent la synthèse d'ADN qui peut être restaurée en rajoutant MCM3 élué du complexe avec l'anticorps. Comme attendu, MCM3 est associé à la chromatine en phase Gl et s'en dissocie durant la réplication, étant absent en phase G2. Ces travaux importants ne sont qu'une étape de la compréhension des mécanismes de la synchronisation entre la synthèse d'ADN et la division cellulaire. En effet, les autres composants responsables de l'activité RLF restent à découvrir, de même que reste à élucider l'action de ces protéines, les rendant indispensables à la synthèse d'ADN.

[1. Chong JPJ, et al. Nature 1995; 375: 418 - 21.]

[2. Madine MA, et al. Nature 1995; 375: 421-4.]

[3. Kubota Y, et al. Cell 1995; 81 : 601-9.]

Les nouveaux partenaires des récepteurs apoptopiques Fas et récepteurs du TNF (TNF-R). L'activation par leurs ligands réceptifs des récepteurs Fas et TNF-R, qui appartiennent à la même famille, aboutit à l'apoptose [1,2], mais on ne connaît pas bien les intermédiaires de cette réaction. L'apoptose induite semble associée à la phosphorylation de résidus tyrosine, sérine et de thréonine, à l'activation de la sphingomyélinase entraînant la libération de céramide et est inhibée par une protéine tyrosine phosphatase PTP-bas [3]. Un motif des récepteurs apoptopiques indispensable à leur fonction est le domaine appelé death domain, situé dans l'extrémité carboxy-terminale intracellulaire des molécules. Quatre équipes, cherchant à cloner des
ADNc codant pour des partenaires du death domain (DD) du récepteur 1 du TNF (TNF-R1) et de la protéine Fas, ont isolé des séquences codant pour trois molécules dénommées TRADD [4] (TNF-R associated death-domain protein), RIP (receptor interacting protein) [5] et FADD (Fas-associated death-domain protein) [6], ce dernier étant caractérisé sous le nom MORT par une autre équipe [7]. Ces trois molécules sont toutes très différentes les unes des autres à l'exception de la présence du domaine DD. D'ailleurs, la technique du double hybride permet de cloner les ADNc codant pour les récepteurs dont le domaine DD est utilisé comme appât [8], confirmant la propension de ces domaines à interagir avec des domaines identiques ou similaires. La surexpression des différentes protéines dont les $\mathrm{ADNc}$ ont été clonés est capable d'induire l'apoptose. FADD/MORT s'associe au motif DD de Fas, TRADD à celui de TNF-R1 alors que RIP s'associe à l'un et l'autre de ces domaines. Ces résultats ne démontrent pas encore qu'il existe une interaction fonctionnelle entre ces différentes protéines, mais révèlent la multiplicité des molécules qui pourraient être impliquées dans le contrôle de l'apoptose. Chacune de ces molécules pourrait avoir des actions spécifiques, comme le suggère le fait que l'apoptose, pour certaines d'entre elles, exige une coopération entre le domaine DD et d'autres régions de la molécule. En fait, ces découvertes ne permettent pas de mieux comprendre le phénomène de l'apoptose mais créent des outils pour l'étudier.

[1. Golstein P. médecine/sciences 1995 ; 11 : 99-104.]

[2. Martinou J. médecine/sciences $1995 ; 11: 367-73$.

[3. Cleveland JL, Ihle JN. Cell 1995; 81 : 479-82.]

[4. Hsu H, et al. Cell 1995 ; 81 : 495504.]

[5. Stanger BZ, et al. Cell $1995 ; 81$ : 513-23.] 
[6. Schinnaiyan AM, et al. Cell 1995 ; 81 : 505-12.]

[7. Boldin MP, et al. J Biol Chem $1995 ; 270$ : 7795-8.]

[8. Plessis A, Camonis JH. médecine/sciences $1994 ; 10$ : I-IX.]

La chaîne $\beta$ du récepteur de l'IL2 joue un rôle régulateur. Le récepteur de l'interleukine 2 est composé d'au moins trois sous-unités différentes, $\alpha, \beta$ et $\gamma$. La chaîne $\gamma$ est fondamentale dans la transduction du signal de l'IL2 comme en témoignent le déficit immunitaire combiné sévère lié à l'X associé à la mutation de son gène chez l'homme $\left(m / s n^{\circ} 2\right.$, vol. $\left.10, p .233\right)$ et la symptomatologie équivalente obtenue par invalidation génique chez la souris $\left(m / s n^{\circ} 1\right.$, vol. $10, p$. 119). La chaîne $\beta$ semble également impliquée dans la transmission du signal et, d'ailleurs, semble lier une autre interleukine, l'IL15. Cette chaîne $\beta$ du récepteur de l'IL2 est présente non seulement à la surface des cellules $\mathrm{T}$, avec une importante augmentation de sa synthèse après stimulation lymphocytaire, mais aussi à celle de nombreuses autres cellules, notamment d'origine hématopoïétique. L'équipe de T.W. Mak (Toronto, Ontario, Canada) [1] vient d'obtenir des souris déficientes en cette sous-unité IL2R $\beta$ par recombinaison homologue. De manière extrêmement surprenante, la symptomatologie des homozygotes n'est pas un déficit immunitaire mais, au contraire, une hyperactivation des lymphocytes $T$, avec hyperprolifération des lymphocytes B et différenciation en plasmocytes, augmentation de la concentration sérique en immunoglobulines Gl et E et présence d'autoanticorps anti-globules rouges, anti-ADN et anti-noyaux. Le traitement des souris avec des anticorps monoclonaux anti-lymphocytes $\mathrm{T} \mathrm{CD} 4^{+}$permet d'obtenir une déplétion en cellules $\mathrm{T}$ auxiliaires, avec amélioration de l'hyperplasie lymphocytaire B, concentration normale en immunoglobulines sériques et absence de manifestations auto-immunes. Les cellules $\mathrm{T}$ auxiliaires $\mathrm{CD}^{+}$hyperactivées sont non fonctionnelles pour coopérer au développement d'une réponse immunitaire normale, qu'elle soit humorale ou cellulaire. Associées à ces anomalies fonctionnelles des populations lymphocytaires, les souris homozygotes développent un syndrome myéloprolifératif qui n'est pas amélioré par la déplétion en lymphocytes $\mathrm{T} \mathrm{CD}^{+}$alors qu'il peut être provoqué chez des animaux naïs par injection des lymphocytes provenant d'animaux IL $2 \mathrm{R} \beta^{-/-}$. Par conséquent, il se pourrait qu'une autre population lymphocytaire fût altérée par le déficit en chaîne IL $2 R \beta$, entraînant ainsi une réponse hyperproliférative des cellules médullaires. Ces résultats indiquent que la chaîne IL2R $\beta$ du récepteur de l'interleukine 2 joue un double rôle : la transmission du signal passant par l'IL2 et d'autres cytokines telles l'IL15 ; une régulation négative du signal d'activation passant par IL2R $\gamma$. Ainsi la signification fonctionnelle de la complexité des récepteurs de cytokines apparaît-elle sous un nouveau jour: non seulement les différentes chaînes des récepteurs peuvent interagir avec des cytokines différentes, mais encore elles peuvent jouer, au sein du complexe, des rôles de co-activateurs ou de modulateurs négatifs.

[1. Suzuki H, et al. Science 1995 ; 268: 1472 -6.]

Un tour de plus dans le sac à malices des virus : inhibition du transport des peptides antigéniques. Les protéines antigéniques intracellulaires sont dégradées en peptides qui sont ensuite transportés par des transporteurs spécifiques TAP dans la lumière du réticulum endoplasmique où ils forment un complexe ternaire avec la $\beta 2$ micro- globuline et les molécules de classe I du complexe majeur d'histocompatibilité, permettant leur transfert à la membrane [1]. Des cellules mutantes en transporteur TAP n'expriment pas spontanément les molécules de classe I liées aux peptides et accumulent la $\beta 2$ microglobuline et la protéine de classe I dans le réticulum endoplasmique. L'addition d'une forte concentration de peptides antigéniques permettant de diffuser dans la lumière du réticulum endoplasmique même en l'absence de protéine TAP fonctionnelle aboutit à l'expression de protéines de classe I à la membrane. Afin d'échapper à la reconnaissance et à l'élimination des cellules infectées par les lymphocytes T cytotoxiques, de nombreux virus interfèrent avec la présentation à la membrane des peptides antigéniques. C'est notamment le cas du virus de l'Herpes simplex HSV. Une protéine codée par un gène d'expression ultra-précoce, ICP47, est responsable de ce blocage de la présentation des peptides viraux dans le contexte des molécules de classe I. Deux équipes internationales, l'une américano-canadienne de Cambridge (MA,USA), Hamilton (Ontario, Canada) et Bethesda (MD, USA) [2] et l'autre américano-franco-allemande de La Jolla (CA, USA), le CHU Necker à Paris et Martinsried, en Allemagne [3], montrent maintenant que ICP47 s'associe au transporteur TAP dont il bloque la fonction. La présentation de l'épitope viral peut être obtenue lorsque les cellules sont transfectées par un vecteur d'expression commandant la synthèse d'une quantité augmentée de transporteur TAP. Ce mécanisme de protection des virus contre la reconnaissance immune n'avait jamais été décrit auparavant.

[1. De la Salle $\mathrm{H}$, et al. médecine/sciences 1994; 10: 1312-4.]

[2. Hill A, et al. Nature 1995; 375; 411-5.]

[3. Früh K, et al. Nature $1995 ; 375$ : 415-8.] 
La phosphatidylinositol-4,5bisphosphate 5-phosphatase en cause dans le syndrome de Lowe. Le syndrome oculo-cérébro-rénal de Lowe est une maladie liée au chromosome $\mathrm{X}$ associant un retard mental sévère, une aréflexie et une hypotonie, divers troubles oculaires (cataracte, glaucome, diminution de l'acuité visuelle) et un dysfonctionnement tubulaire rénal $(\mathrm{m} / \mathrm{s}$ $n^{\circ} 7$, vol. $8, p .742$ ). Il avait été montré que le défaut résidait dans un gène codant pour une protéine identique pour $51 \%$ à l'inositolpolyphosphate 5-phosphatase, enzyme catalysant l'hydrolyse du phosphate en position 5 de plusieurs des inositolpolyphosphates (inositoltriphosphate, tétraphosphate...). Il est montré aujourd'hui que le substrat privilégié de l'enzyme défectueuse est le phosphatidyl inositol 4,5 bisphosphate [1], le substrat de la phospholipase C dont l'hydrolyse aboutit à la transmission de signaux passant par le $\mathrm{Ca}^{2+}$ et la protéine kinase C. Le caractère pléiotrope des anomalies du syndrome de Lowe est probablement en rapport avec le caractère ubiquitaire de l'enzyme dont le déficit pourrait altérer la transmission des signaux dans de nombreuses cellules.

[1. Zhang X, et al. Proc Natl Acad Sci USA 1995 ; 92 : 4853-6.]

Des modèles humains pour la souris lpr... Il faudrait à un lecteur de médecine/sciences beaucoup d'inattention pour ne pas tout savoir des souris lpr, souffrant d'un syndrome lymphoprolifératif associé à des désordres auto-immuns secondaires à une mutation du récepteur Fas, également connu sous les noms d'Apol et de CD95 [1-3]. F. RieuxLaucat et al., du laboratoire d'Alain Fischer, à l'hôpital Necker-Enfants
Malades à Paris (équipe de J.P. de Villartay), décrivent maintenant trois malades souffrant, eux aussi, de syndromes lymphoprolifératifs dus à des mutations du gène $F A S$, associé dans un cas à des manif estations auto-immunes, [4]. Le malade le plus sévèrement atteint a une délétion du gène emportant, notamment, le death domain de la partie intracytoplasmique de Fas [2, 3] alors que les deux autres malades, de la même famille, ont une délétion de deux bases entraînant un décalage de phase de lecture et la synthèse d'une protéine tronquée. C'est l'un de ces deux malades qui avait des manifestations auto-immunes: anémie hémolytique, neutropénie et thrombopénie. L'apoptose des lymphocytes T, provoquée par un anticorps monoclonal anti-Fas activateur de l'apoptose, est totalement supprimée chez le premier malade gravement atteint et est très anormale chez les deux autres. Ces résultats confirment, chez l'homme, le rôle fondamental de l'apoptose relayée par Fas dans le contrôle de la maturation lymphocytaire. En l'absence de cette voie, la sélection négative éliminant les clones auto-réactifs peut être défectueuse, de même que le phénomène de contrôle limitant, dans certains cas, la prolifération des lymphocytes activés.

[1. Kahn A, Briand P. médecine/sciences $1993 ; 9$ : 663-5.]

[2. Golstein P. médecine/sciences 1995; $11: 99-104$.]

[3. Martinou JC. médecine/sciences $1995 ; 11: 367-73$.

[4. Rieux-Laucat F, et al. Science 1995 ; 268: 1347-9.]

Et pourquoi pas une souris à foie humain ? Nos lecteurs connaissent bien le modèle des souris trans- géniques synthétisant de l'urokinase dans leurs hépatocytes, créé par le laboratoire de Ralph Brinster à Philadelphie (PA, USA). Le transgène urokinase est contrôlé par le enhancer du gène de l'albumine et il entraîne des altérations hépatocytaires considérables de telle sorte que les animaux survivants possèdent des foyers d'hépatocytes dépourvus du transgène, marquant un phénomène de délétion génique suivie d'une forte sélection des hépatocytes sièges de cette délétion (m/s $n^{\circ} 2$, vol. 9, p. 230). Brinster et son équipe avaient déjà démontré que l'injection dans la rate de ces animaux transgéniques d'hépatocytes syngéniques préalablement infectés avec un rétrovirus véhiculant le gène de la $\beta$-galactosidase d'Escherichia coli aboutissait au remplacement des hépatocytes transgéniques par les hépatocytes greffés bien aisés à détecter par la coloration de la $\beta$-galactosidase $\left(m / s n^{\circ} 5\right.$, vol. 10, p. 597). De plus en plus fort..., c'est maintenant par des hépatocytes xénogéniques que Rhim et al., toujours de l'équipe de Brinster, remplacent le foie transgénique pour la construction albumine/urokinase [1]. Ce résultat est obtenu en transférant, par croisement, le transgène albumine/urokinase dans la souris immunotolérante nude. Des hépatocytes de rat sont alors injectés dans la rate de ces animaux $n u / n u$ transgéniques, à l'âge de 15 jours. Après huit à dix semaines, les hépatocytes de rat ont proliféré et complètement remplacé les hépatocytes murins, aboutissant à la synthèse de protéines plasmatiques de rat. L'obtention d'un même résultat avec des hépatocytes humains permettrait d'obtenir des souris qui pourraient être extrêmement intéressantes pour les études de pharmacologie et toxicologie.

[1. Rhim JA, et al. Proc Natl Acad Sci USA 1995 ; 92 : 4942-6.] 